\title{
Boundedness of Commutators of Marcinkiewicz Integrals on Nonhomogeneous Metric Measure Spaces
}

\author{
Guanghui Lu and Shuangping Tao \\ College of Mathematics and Statistics, Northwest Normal University, Lanzhou 730070, China \\ Correspondence should be addressed to Shuangping Tao; taosp@nwnu.edu.cn
}

Received 10 October 2015; Accepted 30 November 2015

Academic Editor: Henryk Hudzik

Copyright ( 2015 G. Lu and S. Tao. This is an open access article distributed under the Creative Commons Attribution License, which permits unrestricted use, distribution, and reproduction in any medium, provided the original work is properly cited.

Let $(\mathscr{X}, d, \mu)$ be a metric measure space satisfying the upper doubling condition and geometrically doubling condition in the sense of Hytönen. The aim of this paper is to establish the boundedness of commutator $\mathscr{M}_{b}$ generated by the Marcinkiewicz integral $\mathscr{M}$ and Lipschitz function $b$. The authors prove that $\mathscr{M}_{b}$ is bounded from the Lebesgue spaces $L^{p}(\mu)$ to weak Lebesgue spaces $L^{q}(\mu)$ for $1 \leq p<n / \beta$, from the Lebesgue spaces $L^{p}(\mu)$ to the spaces $\operatorname{RBMO}(\mu)$ for $p=n / \beta$, and from the Lebesgue spaces $L^{p}(\mu)$ to the Lipschitz spaces $\operatorname{Lip}_{(\beta-n / p)}(\mu)$ for $n / \beta<p \leq \infty$. Moreover, some results in Morrey spaces and Hardy spaces are also discussed.

\section{Introduction}

As we know, the Littlewood-Paley operators are playing an important role in harmonic analysis and PDE. The Marcinkiewicz integral is an essential Littlewood-Paley $g$ function. It is firstly introduced by Marcinkiewicz on $\mathbb{R}$ and it is conjectured that it is bounded on $L^{p}([0,2 \pi])$ for any $p \in(1, \infty)$ (see [1]). In 1958, Stein gave the higherdimensional Marcinkiewicz integral (see [2]). Suppose that $\Omega$ is homogeneous of degree zero on $\mathbb{R}^{n}$, for $n \geq 2$, and has mean value zero on the unit sphere $\mathbb{S}^{n-1} ;$ Marcinkiewicz $\mathscr{M}_{\Omega}$ is defined by

$$
\begin{array}{r}
\mathscr{M}_{\Omega}(f)(x) \\
=\left(\int_{0}^{\infty}\left|\int_{|x-y| \leq t} \frac{\Omega(x-y)}{|x-y|^{n-1}} f(y) d \mu(y)\right|^{2} \frac{d t}{t^{3}}\right)^{1 / 2}, \\
x \in \mathbb{R}^{n} .
\end{array}
$$

In [2], Stein proved that if $\Omega \in \operatorname{Lip}_{\alpha}\left(\mathbb{S}^{n-1}\right)$ for some $\alpha \in(0,1]$, then $\mathscr{M}$ was bounded on $L^{p}\left(\mathbb{R}^{n}\right)$ for any $p \in(1,2]$ and also bounded from $L^{1}\left(\mathbb{R}^{n}\right)$ to $L^{1, \infty}\left(\mathbb{R}^{n}\right)$. In 1990 , Torchinsky and Wang established $L^{p}\left(\mathbb{R}^{n}\right)(1<p<\infty)$ boundedness for the commutator generated by $\mathscr{M}_{\Omega}$ and BMO function (see [3]). In 2007, Mo and Lu obtained boundedness of the commutator generated by $\mathscr{M}_{\Omega}$ and $\operatorname{Lip}_{\beta}$ function in [4]. For more results about this operator, we refer the reader to see [5-8].

Let $\mu$ be a nonnegative Radon measure on $\mathbb{R}^{n}$ which satisfies the polynomial growth condition; that is, there exist positive constants $C_{0}$ and $d \in(0, n]$ such that, for all $x \in \mathbb{R}^{n}$ and $r \in(0, \infty), \mu(B(x, 2 r)) \leq C_{0} r^{d}$, where $B(x, r)=\left\{y \in \mathbb{R}^{n}:|x-y|<r\right\}$. The analysis associated with nondoubling measures $\mu$ is proved to play a striking role in solving the long-standing open Painlevé problem by Tolsa in [9]. Obviously, the nondoubling measure $\mu$ with the polynomial growth condition may not satisfy the wellknown doubling condition, which is a key assumption in harmonic analysis on spaces of homogeneous type. Since then, many results from real analysis and harmonic analysis on the classical Euclidean spaces have been extended to the spaces with nondoubling measures satisfying the polynomial growth condition (see [10-14]). The Marcinkiewicz integral operators and commutators have also been discussed widely on the spaces with nondoubling measure (see [15-17]). In 2010, Hytönen introduced a new class of metric measure spaces satisfying both the so-called geometrically doubling and the upper doubling conditions, which are called nonhomogeneous metric measure space in [18]. In particular, in recent years, a lot of classical results have been proved to be still valid if the underlying spaces are replaced by the nonhomogeneous spaces of Hytönen et al. (see $[19,20])$. For 
example, Lin and Yang in [21] obtained the boundedness of Marcinkiewicz integral on nonhomogeneous metric measure spaces. In 2013, Cao and Zhou considered some operators on Morrey spaces over nonhomogeneous metric measure spaces in [22].

In this paper, we will give some estimates for the commutator of Marcinkiewicz integral on the Lebesgue spaces, Lipschitz spaces, $\mathrm{RBMO}(\mu)$ spaces, Morrey spaces, and Hardy spaces on nonhomogeneous metric measure spaces.

To state our main results, we first recall some necessary notions and remarks. The notion of upper doubling metric measure spaces was originally introduced by Hytönen [18] (see also [19]) as follows.

Definition 1. A metric measure space $(\mathscr{X}, d, \mu)$ is said to be upper doubling, if $\mu$ is Borel measure on $\chi$ and there exist a dominating function $\lambda: \mathscr{X} \times(0, \infty) \rightarrow(0, \infty)$ and a positive constant $C_{\lambda}$ such that for each $x \in \mathscr{X}: r \rightarrow \lambda(x, r)$ is nondecreasing and, for all $x \in \mathscr{X}$ and $r \in(0, \infty)$,

$$
\mu(B(x, r)) \leq \lambda(x, r) \leq C_{\lambda} \lambda\left(x, \frac{r}{2}\right) .
$$

Remark 2. (1) Obviously, a space of homogeneous type is a special case of upper doubling spaces, where one can take the dominating function $\lambda(x, r)=\mu(B(x, r))$. Moreover, let $\mu$ be a nonnegative Radon measure on $\mathbb{R}^{n}$ which only satisfies the polynomial growth condition. By taking $\lambda(x, r)=C_{0} r^{d}$, we see that $\left(\mathbb{R}^{n},|\cdot|, \mu\right)$ is also an upper doubling measure space.

(2) It was proved in [20] that there exists another dominating function $\tilde{\lambda}$ related to $\lambda$ satisfying the property that there exists a positive constant $C_{\widetilde{\lambda}}$ such that $\widetilde{\lambda} \leq \lambda, C_{\tilde{\lambda}} \leq$ $C_{\lambda}$ and, for all $x, y \in \chi$ with $d(x, y) \leq \lambda$,

$$
\tilde{\lambda}(x, r) \leq C_{\widetilde{\lambda}} \tilde{\lambda}(y, r) .
$$

Based on this, we always assume that $(\mathscr{X}, d, \mu)$ is a nonhomogeneous metric measure spaces with the dominating function $\lambda$ that satisfies (3).

The following notion of the geometrically doubling condition is well-known in analysis on metric spaces, which was firstly introduced by Coifman and Weiss in [23, pp. 66-67].

Definition 3. A metric space $(\mathscr{X}, d)$ is said to be geometrically doubling, if there exists some $N_{0} \in \mathbb{N}=\{1,2, \ldots\}$ such that, for any ball $B(x, r) \subset \mathscr{X}$, there exists a finite ball covering $\left\{B\left(x_{i}, r / 2\right)\right\}_{i}$ of $B(x, r)$ such that the cardinality of this covering is at most $N_{0}$.

Remark 4. Let $(\mathscr{X}, d)$ be a metric space. Hytönen showed that the following statements are mutually equivalent (see [18]):

(1) $(\mathscr{X}, d)$ is geometrically doubling.

(2) For any $\epsilon \in(0,1)$ and ball $B(x, r) \subset \mathscr{X}$, there exists a finite ball covering $\left\{B\left(x_{i}, \epsilon r\right)\right\}_{i}$ of $B(x, r)$ such that the cardinality of this covering is at most $N_{0} \epsilon^{-n}$. Here and in what follows, $N_{0}$ is as Definition 3 and $n=\log _{2} N_{0}$.

(3) For every $\epsilon \in(0,1)$, any ball $B(x, r) \subset \mathscr{X}$ can contain at most $N_{0} \epsilon^{-n}$ centers $\left\{x_{i}\right\}_{i}$ of disjoint balls with radius $\epsilon r$.
(4) There exists $M \in \mathbb{N}$ such that any ball $B(x, r) \subset \mathscr{X}$ can contain at most $M$ centers $\left\{x_{i}\right\}_{i}$ of disjoint balls $\left\{B\left(x_{i}, r / 4\right)\right\}_{i=1}^{M}$.

Now we recall the notion of the coefficient $K_{B, S}$ introduced by Hytönen (see [18]), which is analogous to the quantity $K_{Q, R}$ introduced by Tolsa (see $[13,14]$ ).

Definition 5. For any two balls $B \subset S$, define

$$
K_{B, S}=1+\int_{2 S \backslash B} \frac{1}{\lambda\left(c_{B}, d\left(x, c_{B}\right)\right)} d \mu(x),
$$

where above and in that follows, for a ball $B=B\left(c_{B}, r_{B}\right)$ and $\rho>0, \rho B=B\left(c_{B}, \rho r_{B}\right) . c_{B}$ is the center of ball $B$.

Remark 6. The following discrete version, $\widetilde{K}_{B, S}$, of $K_{B, S}$ defined in Definition 5, was first introduced by Bui and Duong in nonhomogeneous metric measure spaces (see [24]), which is more close to the quantity $K_{\mathrm{Q}, R}$ introduced by Tolsa [12] in the setting of nondoubling measures. For any two balls $B \subset S$, let $\widetilde{K}_{B, S}$ be defined by

$$
\widetilde{K}_{B, S}:=1+\sum_{k=1}^{N_{B, S}} \frac{\mu\left(6^{k} B\right)}{\lambda\left(c_{B}, 6^{k} r_{B}\right)},
$$

where $r_{B}$ and $r_{S}$, respectively, denote the radius of the balls $B$ and $S$ and $N_{B, S}$ denote the smallest integer satisfying $6^{N_{B, S}} r_{B} \geq$ $r_{S}$. Obviously, $K_{B, S} \leq C \widetilde{K}_{B, S}$. As was pointed by Bui and Duong in [24], in general, it is not true that $K_{B, S} \sim C \widetilde{K}_{B, S}$.

In [25], Zhou and Wang gave the notion of Lipschitz function as follows.

Definition 7. Given $\beta \in(0,1]$, the function $f: \mathscr{X} \rightarrow \mathbb{C}$ satisfies a Lipschitz condition of order $\beta$ provided

$$
\|f\|_{\operatorname{Lip}_{\beta}}=\sup _{x \neq y, x, y \in X} \frac{|f(x)-f(y)|}{\lambda(x, d(x, y))^{\beta / n}}<\infty,
$$

and we claim that $f \in \operatorname{Lip}_{\beta}$.

Remark 8. Lipschitz condition can also be defined by

$$
\|f\|_{\operatorname{Lip}_{\beta}}=\sup _{x \neq y, x, y \in X} \frac{|f(x)-f(y)|}{\lambda(y, d(x, y))^{\beta / n}}<\infty .
$$

See [25].

Let $\alpha, \beta \in(0, \infty)$. A ball $B \subset \mathscr{X}$ is called $(\alpha, \beta)$-doubling if $\mu(\alpha B) \leq \beta \mu(B)$. It was proved in [18] that if a metric measure space $(\mathscr{X}, d, \mu)$ is upper doubling and $\beta>C_{\lambda}^{\log _{2} \alpha}=\alpha^{\nu}$, then, for every ball $B \subset \mathscr{X}$, there exists some $j \in \mathbb{Z}_{+}$such that $\alpha^{j} \beta$ is $(\alpha, \beta)$-doubling. Moreover, let $(\mathscr{X}, d)$ be geometrically doubling and $\beta>\alpha^{n}$ with $n=\log _{2} N_{0}$ and $\mu$ is Borel measure on $\mathscr{X}$ which is finite on bounded sets. In [18] Hytönen also showed that for $\mu$-almost every $x \in \mathscr{X}$ there exist arbitrarily small $(\alpha, \beta)$-doubling balls centered at $x$. Furthermore, 
the radii of their balls may be chosen to be of the form $\alpha^{-j} r$ for $j \in \mathbb{N}$ and any preassigned number $r \in(0, \infty)$. Throughout this paper, for any $\alpha \in(1, \infty)$ and ball $B, \widetilde{B}$ denotes the smallest $\left(\alpha, \beta_{\alpha}\right)$-doubling ball of the form $\alpha^{j} B$ with $j \in \mathbb{Z}_{+}$, where

$$
\beta_{\alpha}=\alpha^{3(\max (n, v))}+30^{n}+30^{\nu}
$$

In what follows, by a doubling ball we mean a $\left(6, \beta_{6}\right)$-doubling ball and $\widetilde{B}^{6}$ is simply denoted by $\widetilde{B}$.

Now we give the definition of Marcinkiewicz integral (see [21]).

Let $K(x, y)$ be a $\mu$-locally integrable function on $\mathscr{X} \times \mathscr{X} \backslash$ $\{(x, x): x \in \mathscr{X}\}$. Assume that there exists a positive constant $C$ such that, for any $x, y \in \mathscr{X}$ with $x \neq y$,

$$
|K(x, y)| \leq C \frac{d(x, y)}{\lambda(x, d(x, y))}
$$

and, for any $x, y, y^{\prime} \in \mathscr{X}$,

$$
\begin{aligned}
& \int_{d(x, y) \geq 2 d\left(y, y^{\prime}\right)}\left[\left|K(x, y)-K\left(x, y^{\prime}\right)\right|\right. \\
& \left.+\left|K(y, x)-K\left(y^{\prime}, x\right)\right|\right] \frac{1}{d(x, y)} d \mu(x) \leq C .
\end{aligned}
$$

The Marcinkiewicz integral $\mathscr{M}(f)$ associated with the above kernel $K(x, y)$ is defined by

$$
\begin{aligned}
& \mathscr{M}(f)(x) \\
& =\left(\int_{0}^{\infty}\left|\int_{d(x, y) \leq t} K(x, y) f(y) d \mu(y)\right|^{2} \frac{d t}{t^{3}}\right)^{1 / 2},
\end{aligned}
$$

$x \in \mathscr{X}$

Definition 9. Let $b \in \operatorname{Lip}_{\beta}(\mu)$ and $K(x, y)$ satisfy (8) and (9). The commutator of Marcinkiewicz $\mathscr{M}_{b}(f)$ is formally defined by

$$
\begin{aligned}
& \mathscr{M}_{b}(f)(x) \\
& =\left(\int_{0}^{\infty}\left|\int_{d(x, y) \leq t} K(x, y)[b(x)-b(y)] f(y) d \mu(y)\right|^{2} \frac{d t}{t^{3}}\right)^{1 / 2}, \\
& x \in X .
\end{aligned}
$$

Obviously, by taking $\lambda(x, r):=C r^{n}$, we see that, in the classical Euclidean space $\mathbb{R}^{n}$, if

$$
K(x, y)=\frac{\Omega(x-y)}{|x-y|^{n-1}}
$$

with $\Omega$ homogeneous of degree zero and $\Omega \in \operatorname{Lip}_{\alpha}\left(\mathbb{S}^{n-1}\right)$ for some $\alpha \in(0,1]$, then $K$ satisfies (8) and (9) and $\mathscr{M}$ as in (10) is just the Marcinkiewicz integral $\mathscr{M}_{\Omega}$ introduced by Stein in [2].

In 2007, Hu et al. introduced a Hörmander-type condition in [8], defined as follows:

$$
\sup _{\substack{\ell>0, y, y^{\prime} \in \mathbb{R}^{d} \\\left|y-y^{\prime}\right| \leq \ell}} \sum_{k=1}^{\infty} k \int_{2^{k} \ell<|x-y| \leq 2^{k+1} \ell}\left[\left|K(x, y)-K\left(x, y^{\prime}\right)\right|+\left|K(y, x)-K\left(y^{\prime}, x\right)\right|\right] \frac{1}{|x-y|} d \mu(x) \leq C .
$$

According to this, we will consider the following condition to replace (13).

$$
\begin{aligned}
& \sup _{\substack{\ell>0, y, y^{\prime} \in X \\
d\left(y, y^{\prime}\right) \leq \ell}} \sum_{k=1}^{\infty} 2^{k \epsilon} \lambda\left(x, 2^{k} \ell\right) \\
& \quad \cdot\left(\frac{1}{\lambda\left(x, 2^{k} \ell\right)} \int_{2^{k} \ell<d(x, y) \leq 2^{k+1} \ell}\left[\left(\left|K(x, y)-K\left(x, y^{\prime}\right)\right|+\left|K(y, x)-K\left(y^{\prime}, x\right)\right|\right) \frac{1}{d(x, y)}\right]^{s} d \mu(x)\right)^{1 / s} \leq C_{s} .
\end{aligned}
$$

Definition 10. Let $1 \leq s<\infty, 0<\epsilon<1$. The kernel $K(x, y)$ is said to satisfy a Hörmander-type condition if there exist $c_{s}>1$ and $C_{s}>0$ such that, for any $x \in \mathscr{X}$ and $\ell>c_{s} d(x, 0)$,
We denote by $\mathscr{H}^{s}$ the class of kernels satisfying this condition. It is obvious that these classes are nested:

$$
\mathscr{H}^{s_{2}} \subset \mathscr{H}^{s_{1}} \subset \mathscr{H}^{1}, \quad 1<s_{1}<s_{2}<\infty .
$$

Now we recall the notion of $\operatorname{RBMO}(\mu)$ (see [18]) as follows.
Definition 11. Let $\rho \in(1, \infty)$. A function $f \in L_{\text {loc }}^{1}(\mu)$ is said to be in the $\operatorname{RBMO}(\mu)$ if there exist a positive constant $C$ and a complex number $f_{B}$ for any balls $B$,

$$
\frac{1}{\mu(\rho B)} \int_{B}\left|f(y)-f_{B}\right| d \mu(y) \leq C,
$$


and that for any balls $B \subset S$,

$$
\left|f_{B}-f_{S}\right| \leq C K_{B, S}
$$

And $\|f\|_{\mathrm{RBMO}(\mu)}$ is defined to be the infimum of the positive constants $C$ in the above inequalities. From [18], it follows that the definition $\operatorname{RBMO}(\mu)$ is independent of the choice of $\rho \in$ $(1, \infty)$.

We begin with recalling some useful properties of $K_{B, S}$ in Definition 5 (see [18]).

Lemma 12. (1) For all balls $B \subset R \subset S$, it holds true that $K_{B, R} \leq$ $K_{B, S}$.

(2) For any $\rho \in[1, \infty)$, there exists a positive constant $C$, depending on $\rho$, such that, for all balls $B \subset S$ with $r_{S} \leq$ $\rho r_{B}, K_{B, S} \leq C$.

(3) For any $\alpha \in(1, \infty)$, there exists a positive constant $\widetilde{C}$, depending on $\alpha$, such that, for all balls $B, K_{B, \widetilde{B}^{\alpha}} \leq \widetilde{C}$.

(4) There exists a positive constant $c$ such that, for all balls $B \subset R \subset S, K_{B, S} \leq K_{B, R}+c K_{R, S}$. In particular, if $B$ and $R$ are concentric, then $c=1$.

(5) There exists a positive constant $\widetilde{C}$ such that, for all balls $B \subset R \subset S, K_{B, R} \leq \widetilde{c} K_{B, S}$; moreover, if $B$ and $R$ are concentric, then $K_{R, S} \leq K_{B, S}$.

The following characterizations of $\operatorname{Lip}_{\beta}(\mu)(0<\beta \leq 1)$ from [25] play a key role in the proofs of theorems.

Lemma 13. For a function $f \in L_{l o c}^{1}(\mu)$, the following conditions (i), (ii), and (iii) are equivalent.

(i) There exist some constant $C_{1}$ and a collection of numbers of $f_{B}$, one for each ball $B$, such that these two properties hold: For any ball $B$ with radius $r$

$$
\frac{1}{\mu(2 B)} \int_{B}\left|f(x)-f_{B}\right| d \mu(x) \leq C_{1} \lambda\left(x_{B}, r_{B}\right)^{\beta / n}
$$

and, for any ball $R$ such that $B \subset R$ and $r_{R} \leq 2 r$,

$$
\left|f_{B}-f_{R}\right| \leq C_{1} \lambda\left(x_{B}, r_{B}\right)^{\beta / n} .
$$

(ii) There is a constant $C_{2}$ such that

$$
|f(x)-f(y)| \leq C_{2} \lambda(x, d(x, y))^{\beta / n},
$$

for $\mu$-almost every $x$ and $y$ in the support of $\mu$.

(iii) For any given $p, 1 \leq p \leq \infty$, there is a constant $C(p)$, such that, for every ball $B$ of radius $r$, we have

$$
\begin{aligned}
& \left(\frac{1}{\mu(B)} \int_{B}\left|f(x)-m_{B}(f)\right|^{p} d \mu(x)\right)^{1 / p} \\
& \quad \leq C(p) \lambda\left(x_{B}, r_{B}\right)^{\beta / n},
\end{aligned}
$$

where $m_{B}=(1 / \mu(B)) \int_{B} f(y) d \mu(y)$ and also for any ball $R$ such that $B \subset R$ and $r_{R} \leq 2 r$

$$
\left|m_{B}(f)-m_{R}(f)\right| \leq C(p) \lambda\left(x_{B}, r_{B}\right)^{\beta / n} .
$$

In addition, the quantities inf $C_{1}, \inf C_{2}$, and inf $C(p)$ with a fixed $p$ are equivalent and denoted by $\|b\|_{L_{i p_{\beta}}(\mu)}$.
Remark 14. For $0<\beta \leq 1$, (20) is equivalent to

$$
\left|f_{B}-f_{R}\right| \leq C_{1}^{\prime} K_{B, S} \lambda(x, r)^{\beta / n},
$$

for any two balls $B \subset R$ with $r_{B} \leq 2 r_{R}$ (see [25]).

The organization of this paper is as follows. In Sections 2 and 3 , we study the commutator $\mathscr{M}_{b}$ in the case of $b \in$ $\operatorname{Lip}_{\beta}(\mu)$ and establish that $\mathscr{M}_{b}$ is bounded from the Lebesgue spaces $L^{p}(\mu)$ to the Lebesgue spaces $L^{q}(\mu)$ for $1 \leq p<$ $n / \beta$, from the Lebesgue spaces $L^{p}(\mu)$ to the spaces $\operatorname{RBMO}(\mu)$ for $p=n / \beta$, and from the Lebesgue spaces $L^{p}(\mu)$ to the Lipschitz spaces $\operatorname{Lip}_{(\beta-n / p)}(\mu)$ for $n / \beta<p \leq \infty$. In Section 4 , we establish the boundedness of commutator of the Marcinkiewicz integral $\mathscr{M}_{b}$ from the Morrey space $M_{q}^{p}(\mu)$ to the Morrey spaces $M_{t}^{s}(\mu)$, from the Morrey spaces $M_{q}^{p}(\mu)$ to the spaces $\operatorname{RBMO}(\mu)$, and from the Morrey spaces $M_{q}^{p}(\mu)$ to the Lipschitz spaces $\operatorname{Lip}_{(\beta-n / p)}(\mu)$. Finally, we establish the boundedness of $\mathscr{M}_{b}$ in $H_{\text {fin }}^{1, \infty, 0}(\mu)$ for $1 / q=1-\beta / n$.

Throughout this paper, we use the constant $C$ with subscripts to indicate its dependence on the parameters. For a $\mu$-measurable set $E, \chi_{E}$ denotes its characteristic function. For any $p \in[1, \infty]$, we denote by $p^{\prime}$ its conjugate index; namely, $1 / p+1 / p^{\prime}=1$

\section{Boundedness of $\mathscr{M}_{b}$ in Lebesgue Spaces}

In this section, we investigate the boundedness of commutator $\mathscr{M}_{b}$ as in (11) in the Lebesgue spaces. The main results are listed as follows.

Theorem 15. Let $b \in \operatorname{Lip}_{\beta}(\mu), 0<\beta \leq 1,1 \leq p<n / \beta$, and $1 / q=1 / p-\beta / n$. Suppose that $K(x, y)$ satisfies (8) and (9), $\mathscr{M}$ is bounded on $L^{2}(\mu)$, and $\mathscr{M}_{b}$ is defined as (11). Then there exists a positive constant $C$ such that, for all $\lambda>0$, one has

$$
\begin{gathered}
\mu\left(\left\{x \in \mathscr{X}: \mathscr{M}_{b}(f)(x)>\lambda\right\}\right) \\
\leq C\left(\frac{\|f\|_{L^{1}(\mu)}}{\lambda}\right)^{n /(n-\beta)} .
\end{gathered}
$$

Proof. By Minkowski inequality and the kernel condition, we deduce that

$$
\begin{gathered}
\mathscr{M}_{b}(f)(x) \leq \int_{X}|K(x, y)||b(x)-b(y)||f(y)| \\
\cdot\left(\int_{d(x, y)}^{\infty} \frac{d t}{t^{3}}\right)^{1 / 2} d \mu(y) \\
\leq C \int_{x} \frac{d(x, y)}{\lambda(x, d(x, y))}|b(x)-b(y)||f(y)| \\
\cdot \frac{1}{d(x, y)} d \mu(y) \leq C\|b\|_{\operatorname{Lip}_{\beta}(\mu)} I_{\beta}(|f|)(x),
\end{gathered}
$$


where $I_{\beta}$ denotes the fractional integral operator defined by

$$
\begin{array}{rl}
I_{\beta} f(x)=\int_{X} f(y)[\lambda(x, d(x, y))]^{(\beta-n) / n} & d \mu(y), \\
& 0<\beta<n .
\end{array}
$$

By applying (2) and Theorem 1.1 in [26], it is easy to get that there exists a positive constant $C>0$, such that, for all $\eta>0$, one has

$$
\mu\left(\left\{x \in \mathscr{X}: I_{\beta}(f)(x)>\eta\right\}\right) \leq C\left(\frac{\|f\|_{L^{1}(\mu)}}{\eta}\right)^{n /(n-\beta)} .
$$

This finishes the proof of Theorem 15.

By applying Marcinkiewicz interpolation theorem, it is easy to get the following result.

Corollary 16. Let $b \in \operatorname{Lip}_{\beta}(\mu), 0<\beta \leq 1,1<p<n / \beta$, and $1 / q=1 / p-\beta / n$. Suppose that $K(x, y)$ satisfies (8) and (9), $\mathscr{M}$ is bounded on $L^{2}(\mu)$, and $\mathscr{M}_{b}$ is defined as (11). Then there exists a positive constant $C$ such that, for all $\lambda>0$,

$$
\left\|M_{b}(f)\right\|_{L^{q}(\mu)} \leq C\|b\|_{L_{i_{\beta}(\mu)}}\|f\|_{L^{p}(\mu)} .
$$

\section{Boundedness of $\mathscr{M}_{b}$ in Lipschitz Spaces}

In this section, we investigate the boundedness of commutator $\mathscr{M}_{b}$ as in (11) in the Lipschitz space.

Theorem 17. Let $0<\epsilon<1,0<\beta<\min \{1 / 2, \epsilon\}, n / \beta<$ $p<\infty$. Suppose that $b \in \operatorname{Lip}_{\beta}(\mu)$ and $K(x, y)$ satisfies (8) and $\mathscr{H}^{p^{\prime}}$ condition. Then $\mathscr{M}_{b}$ is bounded from the Lebesgue spaces $L^{p}(\mu)$ to the Lipschitz spaces $\operatorname{Lip}_{(\beta-n / p)}(\mu)$; namely, there exists a positive constant $C$, such that

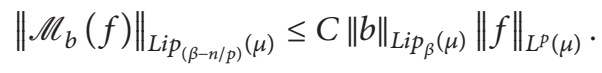

Proof. For any balls $R$ and $S$ in $\mathscr{X}$ such that $R \subset S$ satisfies $r_{S} \leq 2 r_{R}$, let

$$
\begin{aligned}
a_{R} & =m_{R}\left[\mathscr{M}_{b}\left(f \chi_{X \backslash 2 R}\right)\right], \\
a_{S} & =m_{S}\left[\mathscr{M}_{b}\left(f \chi_{X \backslash 2 S}\right)\right] .
\end{aligned}
$$

It is easy to see that $a_{R}$ and $a_{S}$ are real numbers. By Lemma 13, we need to show that there exists a constant $C>0$ such that

$$
\begin{aligned}
& \frac{1}{\mu(2 R)} \int_{R}\left|\mathscr{M}_{b}(f)(x)-a_{R}\right| d \mu(x) \\
& \quad \leq C\|b\|_{\operatorname{Lip}_{\beta}(\mu)}\|f\|_{L^{p}(\mu)} \lambda\left(c_{R}, r_{R}\right)^{\beta / n-1 / p},
\end{aligned}
$$

$$
\left|a_{R}-a_{S}\right| \leq C\|b\|_{\operatorname{Lip}_{\beta}(\mu)}\|f\|_{L^{p}(\mu)} \lambda\left(c_{R}, r_{R}\right)^{\beta / n-1 / p} .
$$

Firstly, let us estimate (31). For a fixed ball $R$ and $x \in R$, decompose $f=f_{1}+f_{2}$, where $f_{1}=f \chi_{2 R}$ and $f_{2}=f-f_{1}$. Write that

$$
\begin{aligned}
& \frac{1}{\mu(2 R)} \int_{R}\left|\mathscr{M}_{b}(f)(x)-a_{R}\right| d \mu(x) \\
& \leq \frac{1}{\mu(2 R)} \int_{R}\left|\mathscr{M}_{b}\left(f_{1}\right)(x)\right| d \mu(x) \\
& \quad+\frac{1}{\mu(2 R)} \int_{R}\left|\mathscr{M}_{b}\left(f_{2}\right)(x)-a_{R}\right| d \mu(x) \\
& =I_{1}+I_{2} .
\end{aligned}
$$

Thus, for $1<p_{1}<n / \beta<p$ and $1 / q_{1}=1 / p_{1}-\beta / n$, by Hölder inequality and Lemma 13 , we can get

$$
\begin{aligned}
I_{1} & \leq \frac{1}{\mu(2 R)}\left(\int_{R}\left|\mathscr{M}_{b}\left(f_{1}\right)(x)\right|^{q_{1}} d \mu(x)\right)^{1 / q_{1}} \\
& \cdot \mu(R)^{1-1 / q_{1}} \leq C \\
& \cdot \frac{\|b\|_{\operatorname{Lip}_{\beta}(\mu)}}{\mu(2 R)}\left(\int_{2 R}|f(x)|^{p_{1}} d \mu(x)\right)^{1 / p_{1}} \mu(R)^{1-1 / q_{1}} \\
& \leq C \frac{\|b\|_{\operatorname{Lip}_{\beta}(\mu)}}{\mu(2 R)}\left(\int_{2 R}|f(x)|^{p} d \mu(x)\right)^{1 / p} \\
& \cdot \mu(2 R)^{1 / p_{1}-1 / p} \mu(R)^{1-1 / q_{1}} \leq C\|b\|_{\operatorname{Lip}_{\beta}(\mu)}\|f\|_{L^{p}(\mu)} \\
& \cdot \lambda\left(c_{R}, r_{R}\right)^{\beta / n-1 / p} .
\end{aligned}
$$

Secondly, let us prove $I_{2}$. For any $x, y \in R$, one has

$$
\begin{aligned}
I_{2} & =\frac{1}{\mu(2 R)} \int_{R} \mid \frac{1}{\mu(R)} \int_{R} \mathscr{M}_{b}\left(f_{2}\right)(x) d \mu(y)-\frac{1}{\mu(R)} \\
& \cdot \int_{R} \mathscr{M}_{b}\left(f \chi_{\mathscr{X} \backslash 2 R}\right)(y) d \mu(y) \mid d \mu(x) \\
& \leq \frac{1}{\mu(R)} \int_{2 R} \int_{R} \mid \mathscr{M}_{b}\left(f_{2}\right)(x) \\
& -\mathscr{M}_{b}\left(f_{2}\right)(y) \mid d \mu(x) d \mu(y) .
\end{aligned}
$$

Now let us estimate $\left|\mathscr{M}_{b}\left(f_{2}\right)(x)-\mathscr{M}_{b}\left(f_{2}\right)(y)\right|$. In order to do this, we write

$$
\begin{aligned}
& D_{1}(x, y)=\left(\int_{0}^{\infty}\left[\int_{d(x, z)<t<d(y, z)}|K(x, z)|\left|b(z)-m_{R}(b)\right|\left|f_{2}(z)\right| d \mu(z)\right]^{2} \frac{d t}{t^{3}}\right)^{1 / 2}, \\
& D_{2}(x, y)=\left(\int_{0}^{\infty}\left[\int_{d(y, z)<t<d(x, z)}|K(y, z)|\left|b(z)-m_{R}(b)\right|\left|f_{2}(z)\right| d \mu(z)\right]^{2} \frac{d t}{t^{3}}\right)^{1 / 2},
\end{aligned}
$$




$$
D_{3}(x, y)=\left(\int_{0}^{\infty}\left[\int_{\substack{d(x, z) \leq t \\ d(y, z) \leq t}}|K(x, z)-K(y, z)|\left|b(z)-m_{R}(b)\right|\left|f_{2}(z)\right| d \mu(z)\right]^{2} \frac{d t}{t^{3}}\right)^{1 / 2}
$$

For $x, y \in R$, we have

$$
\begin{aligned}
& \left|\mathscr{M}_{b}\left(f_{2}\right)(x)-\mathscr{M}_{b}\left(f_{2}\right)(y)\right|=\mid\left(\int_{0}^{\infty}\left[\int_{d(x, z) \leq t} K(x, z)[b(x)-b(z)] f_{2}(z) d \mu(z)\right]^{2} \frac{d t}{t^{3}}\right)^{1 / 2} \\
& \quad-\left(\int_{0}^{\infty}\left[\int_{d(y, z) \leq t} K(y, z)[b(y)-b(z)] f_{2}(z) d \mu(z)\right]^{2} \frac{d t}{t^{3}}\right)^{1 / 2} \mid \\
& \quad \leq\left(\int_{0}^{\infty}\left[\int_{d(x, z) \leq t} K(x, z)[b(x)-b(z)] f_{2}(z) d \mu(z)-\int_{d(y, z) \leq t} K(y, z)[b(y)-b(z)] f_{2}(z) d \mu(z)\right]^{2} \frac{d t}{t^{3}}\right)^{1 / 2} \\
& \quad \leq \sum_{i=1}^{3} D_{i}(x, y)+\left(\int_{0}^{\infty}\left[\int_{d(x, z) \leq t} K(x, z)\left[b(x)-m_{R}(b)\right] f_{2}(z) d \mu(z)\right]^{2} \frac{d t}{t^{3}}\right)^{1 / 2} \\
& \quad+\left(\int_{0}^{\infty}\left[\int_{d(y, z) \leq t} K(y, z)\left[b(y)-m_{R}(b)\right] f_{2}(z) d \mu(z)\right]^{2} \frac{d t}{t^{3}}\right)^{1 / 2} \leq \sum_{i=1}^{3} D_{i}(x, y)+H_{1}+H_{2} .
\end{aligned}
$$

Noticing that, for any $x, y \in R, z \in \mathscr{X} \backslash 2 R$, it holds true that $d(x, z) \geq r_{R}$. By Hölder inequality and Lemma 13, we can deduce that

$$
\begin{aligned}
D_{1}(x, y) & \leq C\left(\int_{0}^{\infty}\left[\int_{d(x, z)<t<d(y, z)} \frac{d(x, z)}{\lambda(x, d(x, z))}\left|b(z)-m_{R}(b)\right|\left|f_{2}(z)\right| d \mu(z)\right]^{2} \frac{d t}{t^{3}}\right)^{1 / 2} \\
& \leq C[d(x, y)]^{1 / 2} \int_{X \mid 2 R} \frac{\left|b(z)-m_{R}(b)\right|}{\lambda(x, d(x, z))}|f(z)| \frac{1}{[d(x, z)]^{1 / 2}} d \mu(z) \\
& \leq C \sum_{k=1}^{\infty} \frac{1}{\lambda\left(c_{R}, 2^{k+1} R\right)} \int_{2^{k} R}\left|b(z)-m_{R}(b)\right||f(z)| d \mu(z) \\
& \leq C\|f\|_{L^{p}(\mu)} \sum_{k=1}^{\infty} \frac{1}{\lambda\left(c_{R}, 2^{k+1} R\right)}\left(\int_{2^{k+1} R}\left|b(z)-m_{R}(b)\right|^{p^{\prime}} d \mu(z)\right)^{1 / p^{\prime}} \\
& \leq C\|b\|_{\operatorname{Lip}_{\beta}(\mu)}\|f\|_{L^{p}(\mu)} \sum_{k=1}^{\infty} \frac{1}{\lambda\left(x, 2^{k+1} r_{R}\right)} \mu\left(2^{k+1} R\right)^{1-1 / p} \lambda\left(x, 2^{k+1} r_{R}\right)^{\beta / n} \leq C\|b\|_{\operatorname{Lip}_{\beta}(\mu)}\|f\|_{L^{p}(\mu)} \lambda\left(c_{R}, r_{R}\right)^{\beta / n-1 / p} .
\end{aligned}
$$

As $z \in \mathscr{X} \backslash 2 R$, we have

$$
\left|b(z)-m_{R}(b)\right| \leq C\|b\|_{\operatorname{Lip}_{\beta}(\mu)} \lambda\left(c_{R}, 2^{k} r_{R}\right)^{\beta / n} .
$$

By a similar argument as in the estimate of $D_{1}(x, y)$, it follows that

$$
D_{2}(x, y) \leq C\|b\|_{\operatorname{Lip}_{\beta}(\mu)}\|f\|_{L^{p}(\mu)} \lambda\left(c_{R}, r_{R}\right)^{\beta / n-1 / p} .
$$

As $0<\beta<\min \{1 / 2, \epsilon\}$, noting that $K(x, y)$ satisfies the condition $\mathscr{H}^{p^{\prime}}$, we have by applying Minkowski inequality

$$
\begin{gathered}
D_{3}(x, y) \leq \int_{X}|K(x, z)-K(y, z)|\left|b(z)-m_{R}(b)\right| \\
\cdot\left|f_{2}(z)\right|\left(\int_{\substack{d(y, z) \leq t \\
d(x, z) \leq t}} \frac{d t}{t^{3}}\right)^{1 / 2} d \mu(z)
\end{gathered}
$$




$$
\begin{aligned}
& \leq C\|b\|_{\operatorname{Lip}_{\beta}(\mu)} \sum_{k=1}^{\infty} \lambda\left(c_{R}, 2^{k} r_{R}\right)^{\beta / n} \int_{2^{k+1} R \backslash 2^{k} R} \mid K(x, z) \\
& -K(y, z) \mid \frac{|f(z)|}{d(y, z)} d \mu(z) \leq C\|b\|_{\operatorname{Lip}_{\beta}(\mu)}\|f\|_{L^{p}(\mu)} \\
& \cdot \sum_{k=1}^{\infty} \lambda\left(c_{R}, 2^{k} r_{R}\right)^{\beta / n} \\
& \cdot\left(\int_{2^{k+1} R \backslash 2^{k} R}[|K(x, z)-K(y, z)|\right. \\
& \left.\left.\frac{1}{d(x, y)}\right]^{q^{\prime}} d \mu(z)\right)^{1 / q^{\prime}} \\
& \leq C\|b\|_{\operatorname{Lip}_{\beta}(\mu)}\|f\|_{L^{p}(\mu)} \sum_{k=1}^{\infty} \lambda\left(c_{R}, 2^{k} r_{R}\right)^{\beta / n-1 / p} \\
& \cdot 2^{-\epsilon} 2^{\epsilon} \lambda\left(x, 2^{k} r_{R}\right)\left(\frac{1}{\lambda\left(c_{R}, 2^{k} r_{R}\right)}\right. \\
& \cdot \int_{2^{k+1} R \backslash 2^{k} R}[|K(x, z)-K(y, z)| \\
& \left.\left.\cdot \frac{1}{d(x, y)}\right]^{q^{\prime}} d \mu(z)\right)^{1 / q^{\prime}} \\
& \leq C\|b\|_{\operatorname{Lip}_{\beta}(\mu)}\|f\|_{L^{p}(\mu)} \lambda\left(c_{R}, r_{R}\right)^{\beta / n-1 / p} .
\end{aligned}
$$

Finally, let us estimate $H_{1}$. For any $x, y \in R, z \in \mathscr{X} \backslash 2 R$, we have

$$
\begin{aligned}
& H_{1} \leq \int_{\mathscr{X} \backslash 2 R}|K(x, z)|\left|b(x)-m_{R}(b)\right||f(z)| \\
& \cdot\left(\int_{d(x, z)}^{\infty} \frac{d t}{t^{3}}\right)^{1 / 2} d \mu(z) \leq C\|b\|_{\operatorname{Lip}_{\beta}(\mu)} \\
& \cdot \lambda\left(x, r_{R}\right)^{\beta / n} \sum_{k=1}^{\infty} \int_{2^{k+1} R \backslash 2^{k} R} \frac{|f(z)|}{\lambda(x, d(x, z))} d \mu(z) \\
& \quad \leq C\|b\|_{\operatorname{Lip}_{\beta}(\mu)} \lambda\left(x, r_{R}\right)^{\beta / n} \sum_{k=1}^{\infty} \frac{1}{\lambda\left(c_{R}, 2^{k+1} r_{R}\right)} \\
& \quad \cdot \int_{2^{k+1} R \backslash 2^{k} R}|f(z)| d \mu(z) \leq C\|b\|_{\operatorname{Lip}_{\beta}(\mu)} \\
& \quad \cdot\|f\|_{L^{p}(\mu)} \lambda\left(c_{R}, r_{R}\right)^{\beta / n-1 / p} .
\end{aligned}
$$

By a similar argument for $H_{1}$, we can get that

$$
H_{2} \leq C\|b\|_{\operatorname{Lip}_{\beta}(\mu)}\|f\|_{L^{p}(\mu)} \lambda\left(c_{R}, r_{R}\right)^{\beta / n-1 / p} .
$$

Combining these estimates, we can conclude

$$
I_{2} \leq C\|b\|_{\operatorname{Lip}_{\beta}(\mu)}\|f\|_{L^{p}(\mu)} \lambda\left(c_{R}, r_{R}\right)^{\beta / n-1 / p} .
$$

Thus, (31) is proved.

Now, let us proceed to show (32). For any balls $R \subset S \subset \mathscr{X}$ with $x \in R$, where $R$ is an arbitrary ball and $S$ is a doubling ball, denote $N_{R, S}+1$ simply by $N$. Write

$$
\begin{aligned}
\mid a_{R}- & a_{S} \mid \\
\leq & \left|m_{R}\left[\mathscr{M}_{b}\left(f \chi_{X \backslash 2^{N} R}\right)\right]-m_{S}\left[\mathscr{M}_{b}\left(f \chi_{X \backslash 2^{N} R}\right)\right]\right| \\
& +\left|m_{R}\left[\mathscr{M}_{b}\left(f \chi_{2^{N} R \backslash 2 R}\right)\right]\right| \\
& +\left|m_{S}\left[\mathscr{M}_{b}\left(f \chi_{2^{N} R \backslash 2 S}\right)\right]\right|=E_{1}+E_{2}+E_{3} .
\end{aligned}
$$

By a similar argument for $I_{2}$, we have

$$
E_{1} \leq C\|b\|_{\operatorname{Lip}_{\beta}(\mu)}\|f\|_{L^{p}(\mu)} \lambda\left(c_{R}, r_{R}\right)^{\beta / n-1 / p} .
$$

As $y \in S, z \in 2^{N} R \backslash 2 R$, it follows that

$$
\begin{aligned}
& M_{b}\left(f \chi_{2^{N} R \backslash 2 S}\right)(y) \\
& \leq C \int_{2^{N} R \backslash 2 S}|K(y, z)[b(y)-b(z)]| \\
& \cdot\left(\int_{d(y, z)}^{\infty} \frac{d t}{t^{3}}\right)^{1 / 2} d \mu(z) \\
& \leq C \int_{2^{N} R \backslash 2 S} \frac{|f(z)|}{\lambda(y, d(y, z))} \mid b(y) \\
& -b(z) \mid d \mu(z) \leq C\|b\|_{\operatorname{Lip}_{\beta}(\mu)}\|f\|_{L^{p}(\mu)} \\
& \cdot \lambda\left(c_{R}, r_{S}\right)^{\beta / n-1} \mu\left(2^{N} R\right)^{1-1 / p} \leq C\|b\|_{\operatorname{Lip}_{\beta}(\mu)} \\
& \cdot\|f\|_{L^{p}(\mu)} \lambda\left(c_{R}, r_{R}\right)^{\beta / n-1 / p} .
\end{aligned}
$$

Therefore, we obtain that

$$
E_{3} \leq C\|b\|_{\operatorname{Lip}_{\beta}(\mu)}\|f\|_{L^{p}(\mu)} \lambda\left(c_{R}, r_{R}\right)^{\beta / n-1 / p} .
$$

With a similar argument to that in the estimate of $E_{3}$, we can get

$$
E_{2} \leq C\|b\|_{\operatorname{Lip}_{\beta}(\mu)}\|f\|_{L^{p}(\mu)} \lambda\left(c_{R}, r_{R}\right)^{\beta / n-1 / p} .
$$

Thus, (32) is proved and this finishes the proof of Theorem 17.

Theorem 18. Let $b \in \operatorname{Lip}_{\beta}(\mu), 0<\beta \leq 1$, and $p=n / \beta$. Suppose that $K(x, y)$ satisfies (9) and $\mathscr{H}^{p^{\prime}}$ condition, $\mathscr{M}$ is bounded on $L^{2}(\mu)$, and $\mathscr{M}_{b}$ is defined as in (11). Then there exists a positive constant $C$ such that, for all bounded functions $f$ with compact support, one has

$$
\left\|\mathscr{M}_{b}(f)\right\|_{R B M O(\mu)} \leq C\|b\|_{\operatorname{Lip}_{\beta}(\mu)}\|f\|_{L^{n / \beta}(\mu)} .
$$

Proof. With a slight change in the proof of Theorem 17, by applying Lemma 13, it is not difficult to get the proof of Theorem 18. We omit the details here. 


\section{Boundedness of $\mathscr{M}_{b}$ in Morrey Spaces}

In this section, we investigate the boundedness for the commutator $\mathscr{M}_{b}$ defined as (11) in the Morrey space $M_{p}^{q}(\mu)$. Before stating our main results, we need to recall the definition of the Morrey space.

Definition 19. Let $k>1$ and $1 \leq q \leq p<\infty$ :

$$
M_{q}^{p}(\mu)=\left\{f \in L_{\mathrm{loc}}^{q}:\|f\|_{M_{q}^{p}(\mu)}<\infty\right\}
$$

where

$$
\|f\|_{M_{q}^{p}(\mu)}=\sup _{B} \mu(k B)^{1 / p-1 / q}\left(\int_{B}|f(x)|^{q} d \mu(x)\right)^{1 / q} .
$$

It is easy to see that $L^{p}(\mu)=M_{p}^{p}(\mu)$ and $M_{q_{1}}^{p} \subset M_{q_{2}}^{p}$ for $1 \leq q_{2} \leq q_{1} \leq p$. If the underlying spaces are replaced by the nonhomogeneous spaces of Tolsa or Euclidean spaces, the definition of Morrey spaces can be seen in [27]. Cao and Zhou proved that the Morrey space is independent of the choice of $k$ (see [22]). Let

Suppose that $\mathbb{Q}=\left\{B \subset \mathscr{X}: B\right.$ is $\left(6, \beta_{6}\right)$-doubling ball $\}$.

$$
\begin{aligned}
& M_{q}^{p}(d)=\left\{f \in L_{\mathrm{loc}}^{q}:\|f\|_{M_{q}^{p}(d)}\right. \\
& \left.=\sup _{B \in \mathscr{Q}} \mu(B)^{1 / p-1 / q}\left(\int_{B}|f|^{q} d \mu\right)^{1 / q}<\infty\right\} .
\end{aligned}
$$

In [22], it was proved that

$$
\begin{gathered}
M_{q}^{p}(d) \approx M_{q}^{p}(\mu), \quad \text { as } 1 \leq q<p<\infty, \\
\beta_{6}>\left(6^{n}\right)^{q p /(p-q)} .
\end{gathered}
$$

Theorem 20. Let $b \in \operatorname{Lip}_{\beta}(\mu)(0<\beta \leq 1), 1<q \leq p<$ $n / \beta, 1<t \leq s<n / \beta$, and $1 / s=1 / p-\alpha / n, t / s=p / q$. Suppose that $K(x, y)$ satisfies (8) and (9), $\mathscr{M}$ is bounded on $L^{2}(\mu)$, and $\mathscr{M}_{b}$ is defined as in (11). Then $\mathscr{M}_{b}$ is bounded from the space $M_{q}^{p}(\mu)$ to the space $M_{t}^{s}(\mu)$; namely, there exists a positive constant $C$ such that

$$
\left\|\mathscr{M}_{b}(f)\right\|_{M_{t}^{s}(\mu)} \leq C\|b\|_{L_{i_{\beta}(\mu)}}\|f\|_{M_{q}^{p}(\mu)} \text {. }
$$

Proof. By Minkowski inequality and the kernel conditions, we can get

$$
\begin{gathered}
\mathscr{M}_{b}(f)(x) \leq \int_{X}|K(x, y)||b(x)-b(y)||f(y)| \\
\cdot\left(\int_{d(x, y)}^{\infty} \frac{d t}{t^{3}}\right)^{1 / 2} d \mu(y) \leq C\|b\|_{\operatorname{Lip}_{\beta}(\mu)} \\
\cdot \int_{\mathscr{X}} \frac{|f(y)|}{\lambda(x, d(x, y))^{1-\beta / n}} d \mu(y) \leq C\|b\|_{\operatorname{Lip}_{\beta}(\mu)} \\
\cdot I_{\beta}(|f|)(x),
\end{gathered}
$$

from the following fact proved in [22]:

$$
\left\|I_{\beta}(f)\right\|_{M_{t}^{s}(\mu)} \leq C\|f\|_{M_{q}^{p}(\mu)}
$$

so we can easily get the proof of Theorem 20 .

The following theorem is adapted from [28].

Theorem 21. Let $b \in \operatorname{Lip}_{\beta}(\mu)(0<\beta \leq 1), 1 \leq q<p$, and $p>n / \beta$. Suppose that $K(x, y)$ satisfies (9) and $\mathscr{H}^{p^{\prime}}$ condition, $M$ is bounded on $L^{2}(\mu)$, and $\mathscr{M}_{b}$ is defined as in (11). Then there exists a positive constant $C$ such that, for all $f \in M_{q}^{p}(\mu)$,

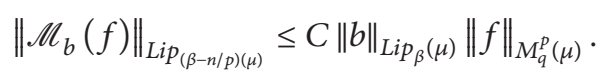

Theorem 22. Let $b \in \operatorname{Lip}_{\beta}(\mu), 0<\beta \leq 1$, and $p=n / \beta$. Suppose that $K(x, y)$ satisfies (9) and $\mathscr{H}^{n /(n-\beta)}$ condition, $\mathscr{M}$ is bounded on $L^{2}(\mu)$, and $\mathscr{M}_{b}$ is defined as in (11). Then there exists a positive constant $C$ such that, for all $f$ with compact support,

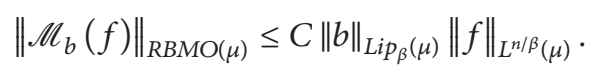

Theorem 23. Let $b \in \operatorname{Lip}_{\beta}(\mu)(0<\beta \leq 1), 1 \leq q<p=$ $n / \beta$. Suppose that $K(x, y)$ satisfies (9) and $\mathscr{H}^{p^{\prime}}$ condition, $\mathscr{M}$ is bounded on $L^{2}(\mu)$, and $\mathscr{M}_{b}$ is defined as in (11). Then there exists a positive constant $C$ such that, for all $f \in M_{q}^{p}(\mu)$,

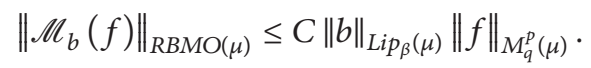

Remark 24. Since $M_{p}^{p}(\mu)=L^{p}(\mu)$, the proof of Theorem 21 is similar to Theorem 17. Theorem 22 can be immediately deduced as a conclusion of Theorem 21 in the case of $p=q=$ $n / \beta$. By applying Lemma 13 , with a slight change in the proof of Theorem 21, it is not difficult to show Theorem 23. Thus, we omit the proofs of Theorems 21-23.

\section{Boundedness of $\mathscr{M}_{b}$ in Hardy Spaces}

This section is devoted to the behavior of $\mathscr{M}_{b}$ in Hardy spaces. In order to define the Hardy space $H^{1}(\mu)$, we need to recall the grand maximal operator $M_{\phi}$ introduced in [14].

Definition 25. Consider $f \in L_{\text {loc }}^{1}(\mu)$, defined by

$$
M_{\phi} f(x)=\sup _{\varphi \sim x}\left|\int_{X} f \varphi d \mu\right|,
$$

where the notation $\varphi \sim x$ means that $\varphi \in L^{1}(\mu) \cap C^{1}(\mathscr{X})$ and satisfies

(1) $\|\varphi\|_{L^{1}(\mu)} \leq 1$,

(2) $0 \leq \varphi(y) \leq 1 / \lambda(y, d(x, y))$ for all $y \in \mathscr{X}$,

(3) $\left|\varphi^{\prime}(y)\right| \leq 1 /[\lambda(y, d(x, y))]^{(n+1) / n}$ for all $y \in \mathscr{X}$.

Based on Theorem 1.2 in [14], we give the following definition of the Hardy space $H^{1}(\mu)$ from [12]. 
Definition 26. The Hardy space $H^{1}(\mu)$ is the set of all functions $f \in L^{1}(\mu)$ satisfying that $\int_{\mathscr{X}} f d \mu=0$ and $M_{\phi} f \in$ $L^{1}(\mu)$. Moreover, the norm of $H^{1}(\mu)$ is defined by

$$
\|f\|_{H^{1}(\mu)}=\|f\|_{L^{1}(\mu)}+\left\|M_{\phi}(\mu)\right\|_{L^{1}(\mu)} .
$$

Now we recall the definition of the atomic Hardy space from $[20]$.

Definition 27. Let $\rho>1$. A function $h \in L_{\text {loc }}^{1}(\mu)$ is called an atomic block if

(1) there exists some ball $B$ such that $\operatorname{supp}(h) \subset B$,

(2) $\int_{X} h(x) d \mu(x)=0$,

(3) for $i=1,2$, there are functions $a_{i}$ supported on cubes $B_{i} \subset S$ and numbers $\lambda_{i} \in \mathbb{R}$ such that $h=\lambda_{1} a_{1}+\lambda_{2} a_{2}$ and

$$
\left\|a_{i}\right\|_{L^{\infty}(\mu)} \leq\left[\mu\left(\rho B_{i}\right) K_{B_{i}, S}\right]^{-1}
$$

Then define

$$
|h|_{H_{\mathrm{atb}}^{1, \infty, 0}(\mu)}=\left|\lambda_{1}\right|+\left|\lambda_{2}\right| .
$$

Define $H_{\text {atb }}^{1, \infty, 0}(\mu)$ and $H_{\text {fin }}^{1, \infty, 0}(\mu)$ as follows:

$$
\begin{aligned}
& \|f\|_{H_{\text {atb }}^{1, \infty, 0}(\mu)}=\inf \left\{\sum_{j}^{\infty}\left|h_{j}\right|_{H_{\text {atb }}^{1, \infty, 0}(\mu)}: f\right. \\
& \left.=\sum_{j=1}^{\infty} h_{j},\left\{h_{j}\right\}_{j \in \mathbb{N}} \text { are }(1, \infty, 0) \text {-atoms }\right\}, \\
& \|f\|_{H_{\text {fin }}^{1, \infty, 0}(\mu)}=\inf \left\{\sum_{j}^{k}\left|h_{j}\right|_{H_{\text {atb }}^{1, \infty, 0}(\mu)}: f\right. \\
& \left.=\sum_{j=1}^{k} h_{j},\left\{h_{j}\right\}_{j=1}^{k} \text { are }(1, \infty, 0) \text {-atoms }\right\},
\end{aligned}
$$

where the infimum is taken over all possible decompositions of $f$ in atomic blocks and $H_{\text {fin }}^{1, \infty, 0}(\mu)$ is the set of all finite linear combinations of $(1, \infty, 0)$-atoms.

Remark 28. It was proved in $[12,20]$ that, for each $\rho>$ 1 , the atomic Hardy space $H_{\mathrm{atb}}^{1, \infty, 0}(\mu)$ is independent of the choice of $\rho$ and the spaces $H^{1}(\mu)$ and $H^{1, \infty, 0}(\mu)$ coincide with equivalent norms.

Then we state the main theorem which was adapted from [28] of this section.

Theorem 29. Let $0<\beta \leq 1, b \in \operatorname{Lip}_{\beta}(\mu)$, and $1 / q=1-$ $\beta / n$. Suppose that $K(x, y)$ satisfies (9) and $\mathscr{H}^{q}$ condition. Then $\mathscr{M}_{b}$ is bounded from the Hardy spaces $H^{1}(\mu)$ into the Lebesgue spaces $L^{q}(\mu)$; namely, there exists a positive constant $C$ such that for all $f \in H_{\text {fin }}^{1, \infty, 0}(\mu)$

$$
\left\|\mathscr{M}_{b}(f)\right\|_{L^{q}(\mu)} \leq C\|b\|_{L_{i p_{\beta}(\mu)}}\|f\|_{H_{f i n}^{1, \infty, 0}(\mu)} .
$$

Proof. Without loss of generality, we may assume that $\rho=$ 4 and $f=\sum h$ as a finite of atomic blocks defined in Definition 27. It is easy to see that we only need to prove the theorem for one atomic block $h$. Let $B$ be a ball such that $\operatorname{supp}(h) \subset B, \int_{\mathscr{X}} h(x) d \mu(x)=0$, and

$$
h(x)=\lambda_{1} a_{1}(x)+\lambda a_{2}(x)
$$

where $\lambda_{i}$, for $i=1,2$, is a real number, $\left|h_{i}\right|_{H_{\text {atb }}^{1, \infty, 0}(\mu)}=\lambda_{1}+\lambda_{2}$, and $a_{i}$, for $i=1,2$, is a bounded function supported on some balls $B_{i} \subset S$ and it satisfies

$$
\left\|a_{i}\right\|_{L^{\infty}(\mu)} \leq\left[\mu\left(4 B_{i}\right) K_{B_{i}, S}\right]^{-1}
$$

Write

$$
\begin{aligned}
\left\|\mathscr{M}_{b}(h)\right\|_{L^{q}(\mu)} \leq & \left(\int_{2 S}\left|\mathscr{M}_{b}(h)(x)\right|^{q} d \mu(x)\right)^{1 / q}+\left(\int_{\mathscr{X} \backslash 2 S}\left|\mathscr{M}_{b}(h)(x)\right|^{q} d \mu(x)\right)^{1 / q} \\
\leq & \left(\int_{2 S}\left|\mathscr{M}_{b}(h)(x)\right|^{q} d \mu(x)\right)^{1 / q} \\
& +\left\{\int_{\mathscr{X} \backslash 2 S}\left(\int_{0}^{d\left(x, x_{S}\right)+2 r_{S}}\left|\int_{d(x, y) \leq t} K(x, y)[b(x)-b(y)] h(y) d \mu(y)\right|^{2} \frac{d t}{t^{3}}\right)^{q / 2} d \mu(x)\right\}^{1 / q} \\
& +\left\{\int_{\mathscr{X} \backslash 2 S}\left(\int_{d\left(x, x_{S}\right)+2 r_{S}}^{\infty}\left|\int_{d(x, y) \leq t} K(x, y)[b(x)-b(y)] h(y) d \mu(y)\right|^{2} \frac{d t}{t^{3}}\right)^{q / 2} d \mu(x)\right\}^{1 / q} \\
= & +I I+I I I .
\end{aligned}
$$


By (67), we have

$$
\begin{aligned}
I \leq & \left|\lambda_{1}\right|\left(\int_{2 S}\left|\mathscr{M}_{b}\left(a_{1}\right)(x)\right|^{q} d \mu(x)\right)^{1 / q} \\
& +\left|\lambda_{2}\right|\left(\int_{2 S}\left|\mathscr{M}_{b}\left(a_{2}\right)(x)\right|^{q} d \mu(x)\right)^{1 / q}=I_{1}+I_{2} .
\end{aligned}
$$

To estimate $I_{1}$, write

$$
\begin{aligned}
I_{1} \leq & \left|\lambda_{1}\right|\left(\int_{6 B_{1}}\left|\mathscr{M}_{b}\left(a_{1}\right)(x)\right|^{q} d \mu(x)\right)^{1 / q} \\
& +\left|\lambda_{1}\right|\left(\int_{2 S \backslash 6 B_{1}}\left|M_{b}\left(a_{1}\right)(x)\right|^{q} d \mu(x)\right)^{1 / q} \\
= & I_{11}+I_{12} .
\end{aligned}
$$

We choose $p_{1}$ and $q_{1}$ such that $1<p_{1}<n / \beta, 1<q<$ $q_{1}$, and $1 / q_{1}=1 / p_{1}-n / \beta$. By Hölder inequality, the fact that $K_{B_{1}, S} \geq 1$, and $\left(L^{p_{1}}(\mu), L^{q_{1}}(\mu)\right)$-boundedness of $\mathscr{M}_{b}$ (see Theorem 15), we have

$$
\begin{aligned}
I_{11} & \leq\left|\lambda_{1}\right|\left[\int_{6 B_{1}}\left|\mathscr{M}_{b}\left(a_{1}\right)(x)\right|^{q_{1}} d \mu(x)\right]^{1 / q_{1}} \\
& \cdot \mu\left(6 B_{1}\right)^{1 / q-1 / q_{1}} \leq C\left|\lambda_{1}\right|\|b\|_{\operatorname{Lip}_{\beta}(\mu)}\left\|a_{1}\right\|_{L^{p_{1}}(\mu)} \\
& \cdot \mu\left(6 B_{1}\right)^{1 / q-1 / q_{1}} \leq C\left|\lambda_{1}\right|\|b\|_{\operatorname{Lip}_{\beta}(\mu)} .
\end{aligned}
$$

Denote $N_{2 B_{1}, 2 S}$ simply by $N_{1}$. Invoking the fact that $\left\|a_{1}\right\|_{L^{\infty}(\mu)} \leq\left[\mu\left(4 B_{1}\right) K_{B_{1}, S}\right]^{-1}$, therefore, we get

$$
\begin{aligned}
I_{12} & \leq\left|\lambda_{1}\right|\left\{\sum_{k=1}^{N_{1}+1} \int_{6^{k+1} B_{1} \backslash 6^{k} B_{1}}\left[\int_{0}^{\infty}\left|\int_{d(x, y) \leq t} K(x, y)[b(x)-b(y)] a_{1}(y) d \mu(y)\right|^{2} \frac{d t}{t^{3}}\right]^{q / 2} d \mu(x)\right\}^{1 / q} \leq C\left|\lambda_{1}\right| \\
& \cdot\left\{\sum_{k=1}^{N_{1}+1} \int_{6^{k+1} B_{1} \backslash 6^{k} B_{1}}\left[\int_{B_{1}} \frac{|b(x)-b(y)|}{\lambda(x, d(x, y))}\left|a_{1}(y)\right| d \mu(y)\right]^{q} d \mu(x)\right\}^{1 / q} \leq C\left|\lambda_{1}\right|\|b\|_{\operatorname{Lip}_{\beta}(\mu)} \\
& \left\{\left\{\sum_{k=1}^{N_{1}+1} \int_{6^{k+1} B_{1} \backslash 6^{k} B_{1}}\left[\int_{B_{1}} \frac{1}{\lambda(x, d(x, y))^{1-\beta / n}}\left|a_{1}(y)\right| d \mu(y)\right]^{q} d \mu(x)\right\}^{1 / q} \leq C\left|\lambda_{1}\right|\|b\|_{\operatorname{Lip}_{\beta}(\mu)}\right. \\
& \cdot\left\{\sum_{k=1}^{N_{1}+1} \lambda\left(x, 6^{k+1} r_{B_{1}}\right)^{q(\beta / n-1)} \mu\left(6^{k+1} B_{1}\right)\left\|a_{1}\right\|_{L^{\infty}(\mu)}^{q} \mu\left(B_{1}\right)^{q}\right\}^{1 / q} \leq C\left|\lambda_{1}\right|\|b\|_{\operatorname{Lip}_{\beta}(\mu)}\left\{K_{B_{1}, S}^{-q} \sum_{k=2}^{N_{1}+1} \frac{\mu\left(6^{k+1} B_{1}\right)}{\lambda\left(x, 6^{k+1} r_{B_{1}}\right)}\right\}^{1 / q}
\end{aligned}
$$

and here we have used the fact that

$$
\sum_{k=2}^{N_{1}+1} \frac{\mu\left(6^{k+1} B_{1}\right)}{\lambda\left(x, 6^{k+1} r_{B_{1}}\right)} \leq C K_{B_{1}, S} .
$$

So we have

$$
I_{1} \leq C\left|\lambda_{1}\right|\|b\|_{\operatorname{Lip}_{\beta}(\mu)}
$$

With an argument similar to that used in the proof of $I_{1}$, we have

$$
I_{2} \leq C\left|\lambda_{1}\right|\|b\|_{\operatorname{Lip}_{\beta}(\mu)}
$$

Combining the estimates for $I_{1}$ and $I_{2}$ yields $I$.

For $i=1,2, y \in B_{i} \subset S, x \in \mathscr{X} \backslash 2 S$, we have $d(x, y) \sim$ $d\left(x, x_{S}\right) \sim d\left(x, x_{S}\right)+2 r_{S}$. By Minkowski inequality, we get

$$
\begin{aligned}
I I & \leq\left\{\int_{\mathscr{X} \backslash 2 S}\left[\int_{X}|K(x, y)||b(x)-b(y)||h(y)|\left(\int_{d(x, y)}^{d\left(x, x_{S}\right)+2 r_{S}} \frac{d t}{t^{3}}\right)^{1 / 2} d \mu(y)\right]^{q} d \mu(x)\right\}^{1 / q} \leq C\|b\|_{\operatorname{Lip}_{\beta}(\mu)} \\
& \cdot \int_{S}\left\{\int_{\mathscr{X} \backslash 2 S}\left(\frac{1}{\lambda(x, d(x, y))^{1-\beta / n}} \frac{r_{S}^{1 / 2}}{[d(x, y)]^{1 / 2}}\right)^{q} d \mu(x)\right\}^{1 / q}|h(y)| d \mu(y) \leq C\|b\|_{\operatorname{Lip}_{\beta}(\mu)}
\end{aligned}
$$




$$
\begin{aligned}
& \cdot \int_{S}\left\{\sum_{k=1}^{\infty} \int_{2^{k+1} S \mid 2^{k} S}\left(\frac{1}{\lambda(x, d(x, y))^{1-\beta / n}} \frac{r_{S}^{1 / 2}}{[d(x, y)]^{1 / 2}}\right)^{q} d \mu(x)\right\}^{1 / q}|h(y)| d \mu(y) \leq C\|b\|_{\operatorname{Lip}_{\beta}(\mu)} \\
& \cdot\left\{\sum_{k=1}^{\infty} \frac{r_{S}^{1 / 2}}{\left(2^{k} r_{S}\right)^{1 / 2}} \frac{1}{\lambda\left(x, 2^{k+1} r_{S}\right)} \mu\left(2^{k+1} S\right)\right\} \int_{S}|h(y)| d \mu(y) \leq C\left(\sum_{j=1}^{2}\left|\lambda_{j}\right|\right) .
\end{aligned}
$$

For any $y \in S$, we have $t \geq d\left(x, x_{S}\right)+2 r_{S} \geq d(x$, $\left.x_{S}\right)+d\left(y, x_{S}\right) \geq d(x, y)$. It follows that

$$
\begin{aligned}
I I I & \leq\left\{\int_{X \backslash 2 S}\left|\int_{S} K(x, y)[b(x)-b(y)] h(y)\left(\int_{d\left(x, x_{S}\right)+2 r_{S}}^{\infty} \frac{d t}{t^{3}}\right)^{1 / 2} d \mu(y)\right|^{q} d \mu(x)\right\}^{1 / q} \\
& \leq C\left\{\int_{X \backslash 2 S}\left|\int_{S} K(x, y)\left[b(x)-m_{S}(b)\right] \frac{h(y)}{d\left(x, x_{S}\right)+2 r_{S}} d \mu(y)\right|^{q} d \mu(x)\right\}^{1 / q} \\
& +C\left\{\int_{\mathscr{X} \backslash 2 S}\left|\int_{S} K(x, y)\left[m_{S}(b)-b(y)\right] \frac{h(y)}{d\left(x, x_{S}\right)+2 r_{S}} d \mu(y)\right|^{q} d \mu(x)\right\}^{1 / q}=I I I_{1}+I I I_{2} .
\end{aligned}
$$

For $I I I_{1}$, by Minkowski inequality, we conclude that

$$
\begin{aligned}
& I I I_{1} \leq C \int_{S} \sum_{k=1}^{\infty}\left(\int_{2^{k+1} S 2^{k} S}\left[\left|K(x, y)-K\left(x, x_{S}\right)\right| \frac{\left|b(x)-m_{S}(b)\right|}{d\left(x, x_{S}\right)+2 r_{S}}\right]^{q} d \mu(x)\right)^{1 / q}|h(y)| d \mu(y) \leq C\|b\|_{\operatorname{Lip}_{\beta}(\mu)} \\
& \cdot \int_{S} \sum_{k=1}^{\infty} 2^{-k \epsilon} 2^{k \epsilon} \lambda\left(c_{S}, 2^{k+1} r_{S}\right)^{1 / q}\left(\frac{1}{\lambda\left(c_{S}, 2^{k+1} r_{S}\right)} \int_{2^{k+1} S 2^{k} S}\left[\lambda\left(x, d\left(x, x_{S}\right)\right)^{\beta / n} \frac{\left|K(x, y)-K\left(x, x_{S}\right)\right|}{d\left(x, x_{S}\right)}\right]^{q} d \mu(x)\right)^{1 / q} \\
& \cdot|h(y)| d \mu(y) \leq C\|b\|_{\operatorname{Lip}_{\beta}(\mu)}\left(\sum_{j=1}^{2}\left|\lambda_{j}\right|\|a\|_{L^{1}(\mu)}\right) \leq C\|b\|_{\operatorname{Lip}_{\beta}(\mu)} \sum_{j=1}^{2}\left|\lambda_{j}\right|,
\end{aligned}
$$

and here we have used the fact that $1 / q=1-\beta / n$ and $0<\epsilon \leq$ 1 .

Now we turn to estimate $\mathrm{III}_{2}$. Noting that, for any $y \in$ $S, x \in \mathscr{X} \backslash 2 S$, we have $d(x, y) \sim d\left(x, x_{S}\right)+2 r_{S}$ and by Minkowski inequality,

$$
\begin{aligned}
& I I I_{2} \\
& \leq C \int_{S} \sum_{k=1}^{\infty}\left(\int_{2^{k+1} S 2_{2^{k} S}}\left[\frac{|K(x, y)|}{d(x, y)}\left|m_{S}(b)-b(y)\right|\right]^{q} d \mu(x)\right)^{1 / q} \\
& \cdot|h(y)| d \mu(y) \\
& \leq C \int_{S} \sum_{k=1}^{\infty}\left(\int_{2^{k+1} S \mid 2^{k} S}\left[\frac{\left|m_{S}(b)-b(y)\right|}{\lambda(x, d(x, y))}\right]^{q} d \mu(x)\right)^{1 / q} \\
& \cdot|h(y)| d \mu(y) \leq C\|b\|_{\operatorname{Lip}_{\beta}(\mu)} \\
& \cdot \int_{S} \sum_{k=1}^{\infty} \lambda\left(c_{S}, r_{S}\right)^{\beta / n} \lambda\left(c_{S}, 2^{k+1} r_{S}\right)^{-1} \mu\left(2^{k+1} S\right)^{1 / q} \\
& \cdot \sum_{j=1}^{2}\left|\lambda_{j}\right|\left|a_{j}(y)\right| d \mu(y) \leq C\|b\|_{\operatorname{Lip}_{\beta}(\mu)} \sum_{j=1}^{2}\left|\lambda_{j}\right| .
\end{aligned}
$$

So we have

$$
I I I \leq C\|b\|_{\operatorname{Lip}_{\beta}(\mu)} \sum_{j=1}^{2}\left|\lambda_{j}\right| .
$$

Combining the estimates for $I, I I$, and $I I I$ yields that

$$
\|\mathscr{M}(h)\|_{L^{q}(\mu)} \leq C|h|_{H_{\mathrm{atb}}^{1, \infty, 0}(\mu)} .
$$

This finishes the proof of Theorem 29.

\section{Conflict of Interests}

The authors declare that there is no conflict of interests regarding the publication of this paper.

\section{Authors' Contribution}

All authors contributed equally to the writing of this paper. All authors read and approved the final paper. 


\section{Acknowledgment}

This paper is supported by National Natural Foundation of China (Grant no. 11561062).

\section{References}

[1] J. Marcinkiewicz, "Sur quelques intégrales du type de Dini," Annales de la Société Polonaise de Mathématique, vol. 17, pp. 4250, 1938.

[2] E. M. Stein, "On the function of Littlewood-Paley, Lusin and Marcinkiewicz," Transactions of the American Mathematical Society, vol. 88, no. 2, pp. 430-466, 1958.

[3] A. Torchinsky and S. Wang, "A note on the Marcinkiewicz integral," Colloquium Mathematicum, vol. 60, no. 1, pp. 235-243, 1990.

[4] H. Mo and S. Lu, "Boundedness of generalized higher commutators of Marcinkiewicz integrals," Acta Mathematica Scientia, vol. 27, no. 4, pp. 852-866, 2007.

[5] Y. Ding, S. Lu, and Q. Xue, "Marcinkiewicz integral on Hardy spaces," Integral Equations and Operator Theory, vol. 42, no. 2, pp. 174-182, 2002.

[6] S. Lu, "Marcinkiewicz integral with rough kernels," Frontiers of Mathematics in China, vol. 3, no. 1, pp. 1-14, 2008.

[7] Y. Ding, Q. Xue, and K. Yabuta, "A remark to the $\mathrm{L}^{2}$ boundedness of parametric Marcinkiewicz integral," Journal of Mathematical Analysis and Applications, vol. 387, no. 2, pp. 691-697, 2012.

[8] G. Hu, H. Lin, and D. Yang, "Marcinkiewicz integrals with nondoubling measures," Integral Equations and Operator Theory, vol. 58, no. 2, pp. 205-238, 2007.

[9] X. Tolsa, "Painlevé's problem and the semiadditivity of analytic capacity," Acta Mathematica, vol. 190, no. 1, pp. 105-149, 2003.

[10] J. García-Cuerva and A. E. Gatto, "Boundedness properties of fractional integral operators associated to non-doubling measures," Studia Mathematica, vol. 162, no. 3, pp. 245-261, 2004.

[11] Y. Sawano and H. Tanaka, "Sharp maximal inequalities and commutators on Morrey spaces with non-doubling measures," Taiwanese Journal of Mathematics, vol. 11, no. 4, pp. 1091-1112, 2007.

[12] X. Tolsa, "BMO, $H^{1}$ and Calderón-Zygmund operators for nondoubling measures," Mathematische Annalen, vol. 319, no. 1, pp. 89-149, 2001.

[13] X. Tolsa, "Littlewood-paley theory and the T(1)theorem with non-doubling measures," Advances in Mathematics, vol. 164, no. 1, pp. 57-116, 2001.

[14] X. Tolsa, "The space $H^{1}$ for nondoubling measures in terms of a grand maximal operator," Transactions of the American Mathematical Society, vol. 355, no. 1, pp. 315-348, 2003.

[15] W. Chen, Y. Meng, and D. Yang, "Calderón-Zygmund operators on Hardy spaces without the doubling condition," Proceedings of the American Mathematical Society, vol. 133, no. 9, pp. 26712680, 2005.

[16] W. Chen and E. Sawyer, "A note on commutators of fractional integral with $\operatorname{RBMO}(\mu)$ functions," Illinois Journal of Mathematics, vol. 46, no. 4, pp. 1287-1298, 2002.

[17] J. Zhou and B. Ma, "Marcinkiewicz commutators with Lipschitz functions in non-homogeneous spaces," Canadian Mathematical Bulletin, vol. 55, no. 3, pp. 646-662, 2012.
[18] T. Hytönen, "A framework for non-homogeneous analysis on metric spaces, and the RBMO space of Tolsa," Publicacions Matemàtiques, vol. 54, no. 2, pp. 485-504, 2010.

[19] T. Hytönen, S. Liu, D. Yang, and D. Yang, "Boundedness of Calderón-Zygmund operators on non-homogeneous metric measure spaces," Canadian Journal of Mathematics, vol. 64, no. 4, pp. 892-923, 2012.

[20] T. Hytönen, D. Yang, and D. Yang, "The Hardy space $H^{1}$ on nonhomogeneous metric spaces," Mathematical Proceedings of the Cambridge Philosophical Society, vol. 153, no. 1, pp. 9-31, 2012.

[21] H. Lin and D. Yang, "Equivalent boundedness of Marcinkiewicz integrals on non-homogeneous metric measure spaces," Science China Mathematics, vol. 57, no. 1, pp. 123-144, 2014.

[22] Y. Cao and J. Zhou, "Morrey spaces for nonhomogeneous metric measure spaces," Abstract and Applied Analysis, vol. 2013, Article ID 196459, 8 pages, 2013.

[23] R. Coifman and G. Weiss, Analyse Harmonique NonCommutative sur Certains Espaces Homogenes, vol. 242 of Lecture Notes in Mathematicas, Springer, Berlin, Germany, 1971.

[24] T. A. Bui and X. T. Duong, "Hardy spaces, regularized BMO and the boundedness of Caldeŕn-Zymgund operators on homogeneous spaces," Journal of Geometric Analysis, vol. 23, no. 2, pp. 895-932, 2013.

[25] J. Zhou and D. Wang, "Lipschitz spaces and fractional integral operators associated with nonhomogeneous metric measure spaces," Abstract and Applied Analysis, vol. 2014, Article ID 174010, 8 pages, 2014.

[26] I. Sihwaningrum and Y. Sawano, "Weak and strong type estimates for fractional integral operators on Morrey spaces over measure spaces," Eurasian Mathematical Journal, vol. 4, no. 1, pp. 76-81, 2013.

[27] Y. Sawano and H. Tanaka, "Morrey spaces for non-doubling measures," Acta Mathematica Sinica, vol. 21, no. 6, pp. 15351544, 2005.

[28] L. Li, "Estimates for Marcinkiewicz commutators with Lipschitz functions under nondoubling measures," Journal of Inequalities and Applications, vol. 2013, article 388, 2013. 


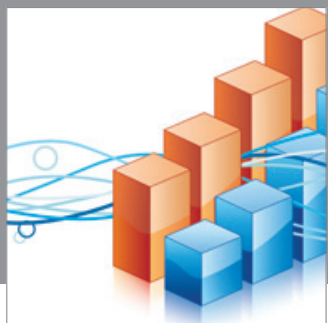

Advances in

Operations Research

mansans

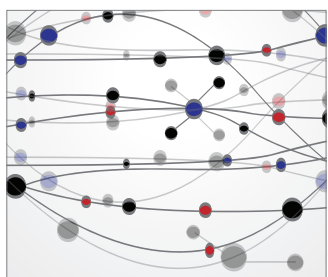

The Scientific World Journal
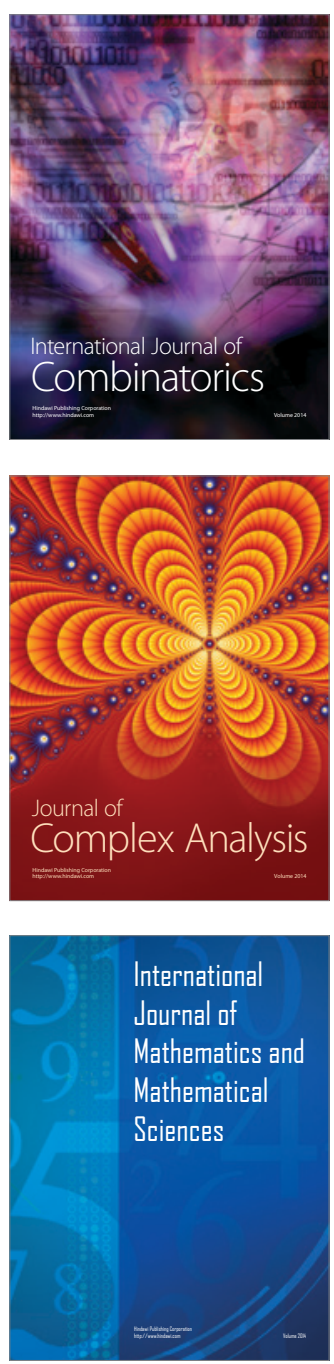
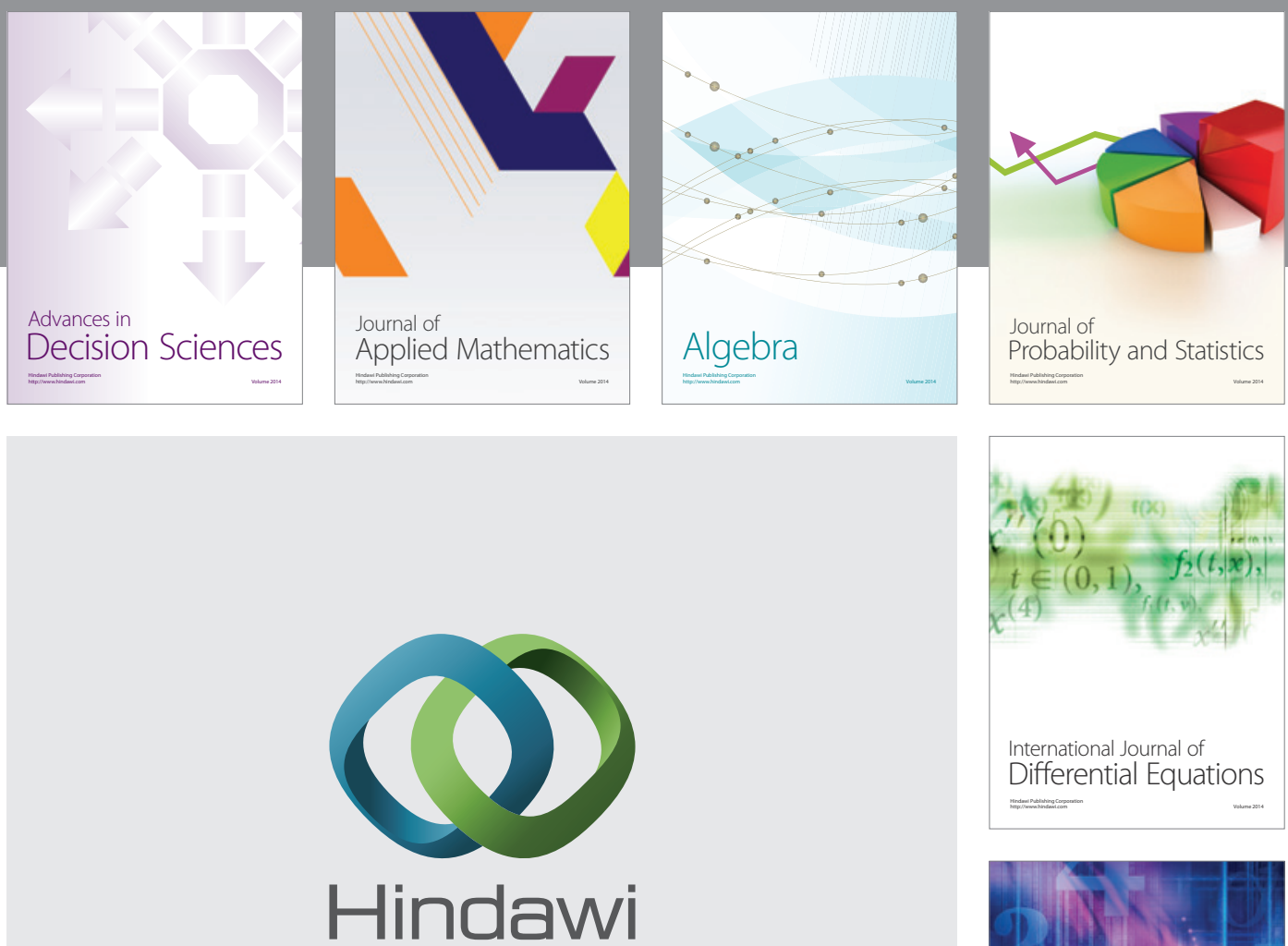

Submit your manuscripts at http://www.hindawi.com
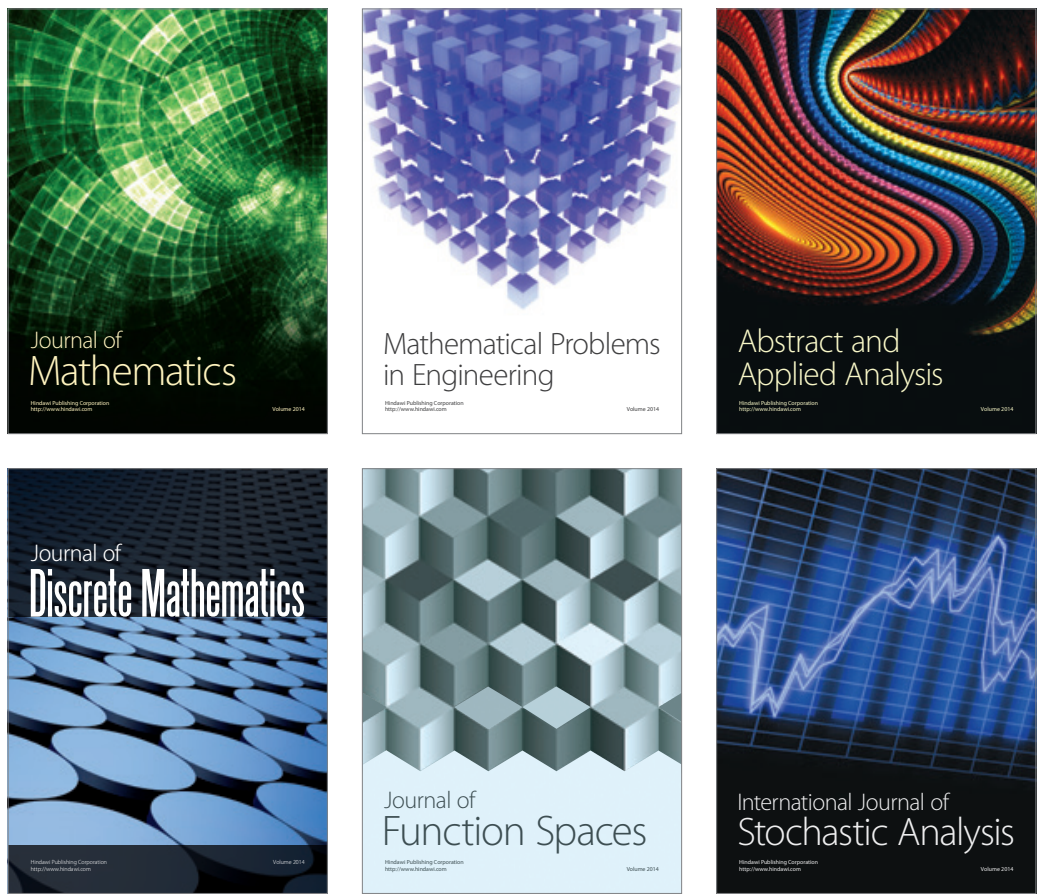

Journal of

Function Spaces

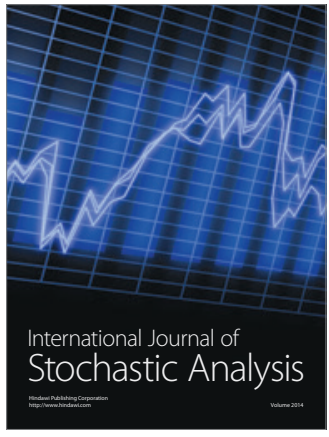

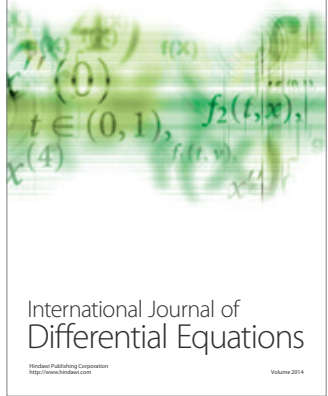
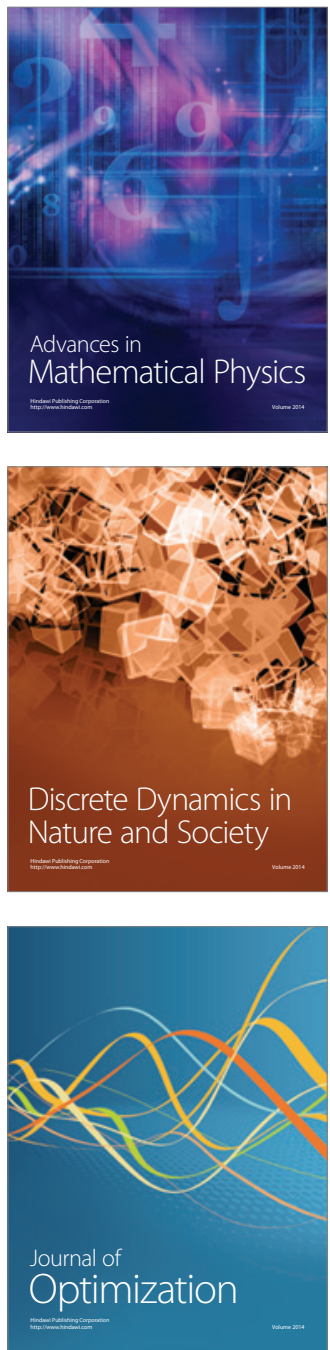